\title{
Anticancer activities of sulindac in prostate cancer cells associated with c-Jun NH2-terminal kinase 1/ $\beta$-catenin signaling
}

\author{
JUN DU $^{1 *}$, YONGCHEN GUO $^{2 *}$, YONGHUA BAO $^{3}$, MENGTAO XING $^{4}$, ABEER M. MAHMOUD $^{4}$, \\ ZHENYONG CHE ${ }^{5}$, ZHIGUO $\mathrm{CHEN}^{6}$ and WANCAI YANG ${ }^{4,6}$ \\ ${ }^{1}$ Department of Urology, Xinxiang Central Hospital, The Teaching Hospital of Xinxiang Medical University, Xinxiang, \\ Henan 453000; Departments of ${ }^{2}$ Laboratory Medicine and ${ }^{3}$ Microbiology, Xinxiang Medical University, Xinxiang, \\ Henan 453003, P.R. China; ${ }^{4}$ Department of Pathology, University of Illinois at Chicago, Chicago, IL 60612, USA; \\ ${ }^{5}$ Department of Neurology, Xinxiang Central Hospital, The Teaching Hospital of Xinxiang Medical University, \\ Xinxiang, Henan 453000; ${ }^{6}$ Department of Pathology, Xinxiang Medical University, \\ Xinxiang, Henan 453003, P.R. China
}

Received July 30, 2013; Accepted March 20, 2014

DOI: $10.3892 / \mathrm{ol} .2014 .2084$

\begin{abstract}
The non-steroidal anti-inflammatory agent, sulindac, has shown strong effects on cancer prevention in colorectal cancers, however, its anticancer activities on prostate cancer remain unclear. In the current study, human prostate cancer cell lines, LNCaP and PC-3, were treated with various concentrations of sulindac and it was found that sulindac significantly inhibits prostate cancer cell proliferation and promotes cell apoptosis in a dose- and time-dependent manner. Further studies revealed that sulindac significantly induces c-Jun NH2-terminal kinase (JNK) 1 phosphorylation and inhibits $\beta$-catenin at the transcriptional and post-transcriptional levels. In conclusion, by targeting the JNK $1 / \beta$-catenin signaling pathway, sulindac may present a potential preventive or therapeutic agent for treatment of patients with prostate cancer.
\end{abstract}

\section{Introduction}

Prostate cancer is one of the most common malignant diseases and the second leading cause of cancer mortality among males

Correspondence to: Dr Yongchen Guo, Department of Laboratory Medicine, Xinxiang Medical University, 601 East Jinsui Avenue, Xinxiang, Henan 453003, P.R. China

Email: guoyongchen2005@126.com

Professor Wancai Yang, Department of Pathology, University of Illinois at Chicago, 840 South Street, Chicago, IL 60612, USA

Email: wyang06@uic.edu

*Contributed equally

Abbreviations: JNK1, c-Jun NH2-terminal kinase 1

Key words: sulindac, prostate cancer, c-Jun NH2-terminal kinase 1, $\beta$-catenin in the USA (1). Sulindac is a non-steroidal anti-inflammatory agent, which has shown significant activity in inhibiting gastrointestinal tumor formation in mouse models of colorectal cancers, as well as in inhibiting colorectal cancer cell proliferation and inducing cell apoptosis $(2,3)$. However, these activities require $\mathrm{p} 21$, not $\mathrm{p} 27(4,5)$, and have been associated with c-Jun NH2-terminal kinase (JNK) 1 activation via phosphorylation in vitro and in vivo (6-8). The current study identified that sulindac exerts anticancer activities on prostate cancer cells via the inhibition of cell proliferation and induction of apoptosis by targeting the JNK1/ $\beta$-catenin signaling pathway.

The JNKs have been identified as members of the mitogen-activated protein kinase family, and phosphorylate and activate various transcriptional factors, including c-Jun, activating transcription factor 2 , activation protein-1 and p53 (9-13). Our previous studies demonstrated that JNK1 is critical in intestinal tumorigenesis, which was identified to be associated with p21 expression in a JNK1 gene knockout mouse model as well as colorectal cancers (14). It is also well known that the JNK signaling transduction pathway is significant in a variety of cellular processes, including cell proliferation, differentiation and apoptosis $(15,16)$, particularly in regulating apoptosis (17-19).

To elucidate the bioactivities of sulindac and the underlying mechanism, the current study analyzed the efficacy of sulindac with regard to dosage as well as the involvement and roles of $\mathrm{JNK} 1 / \beta$-catenin signaling in prostate cancer.

\section{Materials and methods}

Human prostate cancer cell culture. Human prostate cancer cell lines, PC-3 and LNCaP, were purchased from the American Type Culture Collection (Manassas, VA, USA) and maintained in RPMI-1640 media supplemented with $10 \%$ fetal bovine serum (FBS), 1X antibiotic/antimycotic (100 U/ml streptomycin, $100 \mathrm{U} / \mathrm{ml}$ penicillin and $0.25 \mu \mathrm{g} / \mathrm{ml}$ amphotericin B), $100 \mu \mathrm{M}$ non-essential amino acids and $10 \mathrm{mM}$ HEPES buffer solution (all Invitrogen Life Technologies, Carlsbad, CA, USA). 
All cells were cultured at $37^{\circ} \mathrm{C}$ in a humidified atmosphere of $5 \% \mathrm{CO}_{2}$. Sulindac (Sigma-Aldrich, St. Louis, MO, USA) was dissolved in dimethyl sulfoxide (DMSO) and diluted in a serials concentration.

Apoptosis analysis. The treated cells were harvested at different time points, washed in cold phosphate-buffered saline (PBS) and stained with Annexin V and propidium iodide according to the manufacturer's instructions for the Alexa Fluor 488 Annexin V/Dead Cell Apoptosis kit (Invitrogen Life Technologies). The cells were analyzed using a CyAn ADP three channel flow cytometer and Summit3 software (both Beckman Coulter, Miami, FL, USA). The reactions were performed in triplicate and the data are representative of three independent experiments.

Cell proliferation assay. A total of $1 \times 10^{4}$ cells in $100 \mu 1$ RPMI-1640 medium supplemented with 10\% FBS was seeded in 96-well plates one day prior to the assay. After $18-20 \mathrm{~h}$, the medium was removed and $100 \mu \mathrm{l}$ complete assay medium was added to each well and simultaneously, sulindac was added to the medium to reach final concentrations of sulindac; $0,0.4$ and $0.8 \mathrm{mM}$. Next, $100 \mu \mathrm{l}$ full medium with an equal volume of DMSO was added to each well as a control. All of the groups of cells were cultured in triplicate. The plates were incubated at $37^{\circ} \mathrm{C}$ for $24 \mathrm{~h}$ and the cell proliferation was determined by 3-(4,5-dimethyl thiazol-2-yl)-2,5-diphenyl tetrazolium bromide (MTT) assay (CellTiter 96 Non-Radioactive Cell Proliferation Assay kit; Promega Corporation, Madison, WI, USA). Briefly, $15 \mu 1$ MTT (Promega Corporation) was added to each well and the plate was incubated at $7^{\circ} \mathrm{C}$ for $4 \mathrm{~h}$ in a humidified atmosphere of 5\% $\mathrm{CO}_{2}$. Next, $100 \mu 1$ stop solution was added to each well and incubated for $1 \mathrm{~h}$. Finally, the absorbance was measured at $570 \mathrm{~nm}$ using a microplate reader (Synergy 2; BioTek Instruments, Inc., Winooski, VT, USA).

TOP/FOP-Flash transfection and luciferase assay. To examine the effect of sulindac on $\beta$-catenin/T-cell factor(TCF) signaling, cells were seeded in 24 well-plates at a cell density of 5,000 cells/well in minimum essential media (Invitrogen Life Technologies) without antibiotics. The cells were transiently cotransfected with a TOP- or FOP-Flash plasmid (Upstate Biotechnology, Inc., Lake Placid, NY, USA) and the Renilla luciferase expression vector served as a control for transfection efficiency using Lipofectamine 2000 (Invitrogen Life Technologies). At $6 \mathrm{~h}$ after transfection, the medium was removed and the cells were supplied with fresh medium supplemented with $0.8 \mathrm{mM}$ sulindac for $48 \mathrm{~h}$ and DMSO served as a control. The cells were washed with cold PBS, lysed with passive lysis buffer and luciferase activity was measured using the Dual Luciferase Report Assay System (Promega Corporation). The lysate firefly luciferase values were normalized to Renilla luciferase activity and all experiments were independently performed in triplicate.

Immunoblotting analysis. Total protein was isolated from sulindac-treated cells, quantified by Bradford analysis and measured at $595 \mathrm{~nm}$ with a microplate reader using the Bio-Rad Protein Assay kit (Bio-Rad, Hercules, CA, USA).
Next, $30 \mu \mathrm{g}$ protein/lane was resolved by $10 \%$ SDS-PAGE and transferred to polyvinylidene fluoride membranes (Millipore, Bedford, MA, USA). The immunoblot was incubated overnight at $4^{\circ} \mathrm{C}$ with the primary antibodies, anti-JNK1 and -phosphorylated-JNK1 (p-JNK) purchased from Cell Signaling Technology, Inc. (Danvers, MA, USA). $\beta$-catenin was obtained from Sigma-Aldrich and horseradish peroxidase-conjugated affinipure goat anti-mouse IgG (Promega Corporation) secondary antibodies were used. Electrochemiluminescence western blotting detection reagents (Amersham Pharmacia Biotech, Piscataway, NJ, USA) were used as the protein signal and $\beta$-actin (Sigma-Aldrich) served as a loading control.

\section{Results}

Sulindac induces human prostate cancer apoptosis. To determine the effects of sulindac on prostate cancer cell apoptosis, PC-3 and LNCaP cells were treated with various concentrations of sulindac $(0,0.4$ and $0.8 \mathrm{mM})$ for $48 \mathrm{~h}$. Sulindac was found to significantly induce cell apoptosis in the two cell lines (Fig. 1 A; $\mathrm{P}<0.05$ at $0.4 \mathrm{mM}$ and $\mathrm{P}<0.01$ at $0.8 \mathrm{mM}$ ) compared with the untreated groups. To determine if the apoptosis was induced in a time-dependent manner, the cells were treated with $0.8 \mathrm{mM}$ sulindac for 24 and $48 \mathrm{~h}$. As shown in Fig. 1B, following $24 \mathrm{~h}$ of treatment, sulindac was found to promote apoptosis in the PC-3 and LNCaP cells $(\mathrm{P}<0.05)$, however, following $48 \mathrm{~h}$ of treatment, the induction of apoptosis was more significant $(\mathrm{P}<0.01)$ compared with the untreated groups. These results indicated that sulindac induces apoptosis in a dose- and time-dependent manner.

Sulindac inhibits human prostate cancer cell proliferation. Next, the effects of sulindac on prostate cancer cell proliferation were determined. As shown in Fig. 2A, $0.8 \mathrm{mM}$ sulindac was found to significantly inhibit cell proliferation in the PC-3 and $\mathrm{LNCaP}$ cell lines $(\mathrm{P}<0.05)$. However, $0.4 \mathrm{mM}$ sulindac also inhibited cell proliferation in the two cell lines $(\mathrm{P}>0.05)$ in comparison with the untreated groups as determined by MTT. In addition, $0.8 \mathrm{mM}$ sulindac was found to significantly inhibit cell proliferation following $48 \mathrm{~h}$ treatment $(\mathrm{P}<0.05)$ compared with the untreated groups (Fig. 2B). These results indicated that cell proliferation inhibition by sulindac occurred in a dose- and time-dependent manner.

Sulindac inhibits $\beta$-catenin/TCF signaling in human prostate cancer cells. Our previous studies demonstrated that sulindac inhibits colorectal cancer cell proliferation via Wnt- $\beta$-catenin/TCF signaling (6). In the current study, it was determined that sulindac exhibits similar mechanisms within prostate cancer cells. Cells were transiently transfected with a $\beta$-catenin-TCF luciferase reporter construct, TOP-Flash, which contains multiple optimal TCF/lymphocyte enhancing factor (LEF) binding sites that induce transcription of a luciferase reporter gene when activated by $\beta$-catenin, or a negative control FOP-Flash, which contains mutant and inactivated TCF/LEF binding sites. As shown in Fig. 3, sulindac was found to inhibit $\beta$-catenin-TCF luciferase reporter activities by $\sim 40 \%$ in PC-3 cells and $\sim 50 \%$ in LNCaP cells $(\mathrm{P}<0.05)$ compared with the untreated cells. 
A

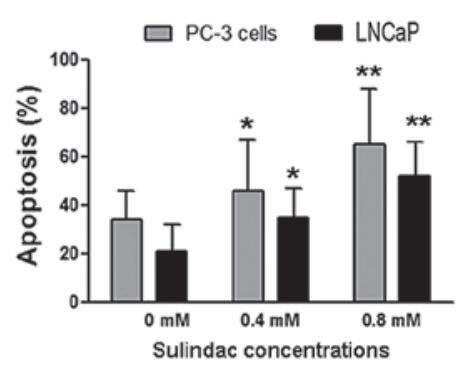

B $\square$ PC-3 $(0.8 \mathrm{mM})$

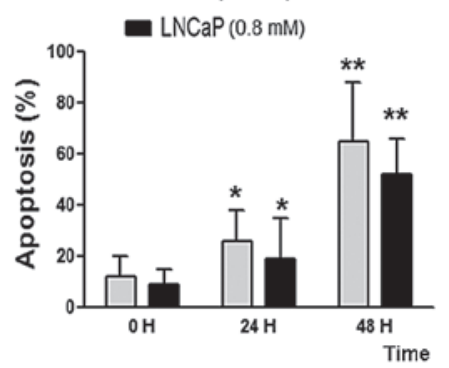

Figure 1. Sulindac induces human prostate cancer cell apoptosis in a dose- and time-dependent manner (human prostate cancer cell lines, LNCaP and PC-3). Cells treated with (A) 0-0.8 mM sulindac for $48 \mathrm{~h}$ and (B) $0.8 \mathrm{mM}$ sulindac for $0-48 \mathrm{~h}$ were analyzed by flow cytometry. ${ }^{*} \mathrm{P}<0.05$; ${ }^{* *} \mathrm{P}<0.01 \mathrm{vs}$. the non-treatment groups.

A

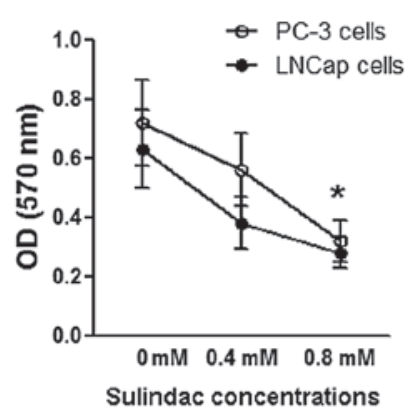

$\mathbf{B}$

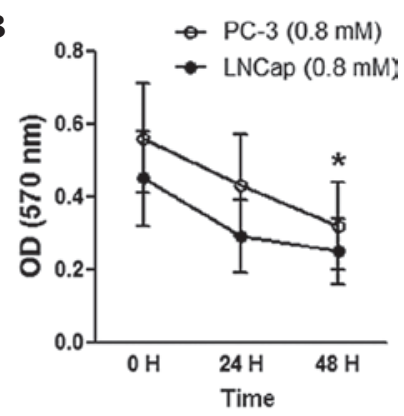

Figure 2. Sulindac inhibits human prostate cancer cell proliferation in a dose- and time-dependent manner (human prostate cancer cell lines, LNCaP and PC-3). Cells treated with (A) 0-0.8 mM sulindac for $48 \mathrm{~h}$ and (B) $0.8 \mathrm{mM}$ sulindac for 0-48 h were analyzed by 3-(4,5-dimethyl thiazol-2-yl)-2,5-diphenyl tetrazolium bromide assay ${ }^{*} \mathrm{P}<0.05$ vs. the non-treatment groups. OD, optical density.

A

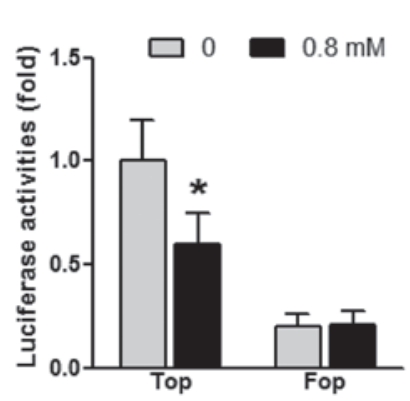

B

LNCap cells

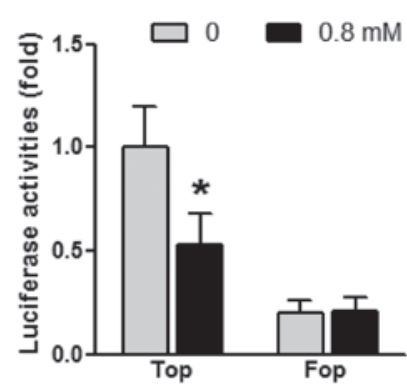

Figure 3. Sulindac inhibits Wnt $\beta$-catenin at the transcriptional level as measured by analyzing the TOP/FOP-Flash luciferase activities in human prostate cancer cells. (A) PC-3 and (B) LNCaP cells cotransfected with TOP- or FOP-Flash and Renilla were treated with $0.8 \mathrm{mM}$ sulindac for $48 \mathrm{~h}$ and harvested for luciferase analysis. FOP-Flash served as a negative control and Renilla served as an internal control. ${ }^{*} \mathrm{P}<0.05$ vs. untreated cells.

Sulindac suppresses $\beta$-catenin expression and induces JNK1 phosphorylation in human prostate cancer cells. To determine the mechanism of the sulindac-mediated induction of apoptosis and inhibition of cell proliferation in prostate cancer cells, the changes in $\beta$-catenin and JNK1 phosphorylation were investigated. Following $48 \mathrm{~h}$ of treatment, $0.8 \mathrm{mM}$ sulindac was found to suppress $\beta$-catenin expression and induce JNK1 phosphorylation (an activated form of JNK1) in the PC-3 and LNCaP cells (Fig. 4).

\section{Discussion}

Previous studies have shown the cancer preventive activities of sulindac on gastrointestinal cancers $(4,5)$. The current

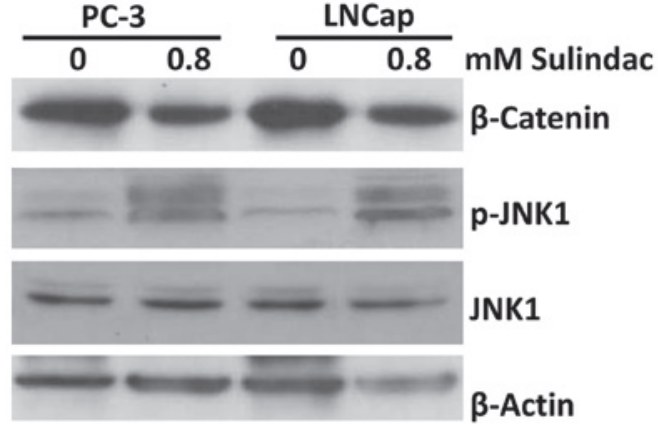

Figure 4. Sulindac suppresses $\beta$-catenin expression and induces JNK1 phosphorylation in human prostate cancer cells. Cells were treated with $0.8 \mathrm{mM}$ sulindac for $48 \mathrm{~h}$ and harvested for protein extraction for immunoblotting. $\beta$-actin served as an internal control. JNK1, c-Jun NH2-terminal kinase 1. 
study demonstrated that sulindac also exhibits an anticancer function within human prostate cancer cells via the promotion of cancer cell apoptosis and inhibition of cell proliferation, which was associated with the suppression of $\beta$-catenin/TCF signaling and increased JNK1 phosphorylation.

In addition to the preventive effect of sulindac on colorectal cancer, sulindac also exerts tumor inhibition on human lung and breast cancer cells (6). The present study provides evidence that sulindac influences cancer inhibition in prostate cancer cells. Similar to colorectal, lung and breast cancer, in prostate cancer cells, $\beta$-catenin is highly expressed and may present a therapeutic target for sulindac. Furthermore, sulindac was found to significantly suppress $\beta$-catenin expression at the translational and transcriptional levels, as determined by the inhibition of TOP-Flash, a vector containing multiple optimal $\mathrm{TCF} / \mathrm{LEF}$ binding sites that induce transcription of a luciferase reporter gene when $\beta$-catenin is activated.

Our previous studies demonstrated that $\beta$-catenin is negatively regulated by p-JNK1 in colorectal cancers $(20,21)$. The current study also showed that the suppression of $\beta$-catenin by sulindac is associated with increased levels of p-JNK1, although total JNK1 levels were not changed, which provides increased evidence of the involvement of sulindac in tumor inhibition by targeting the JNK1/ $\beta$-catenin signaling pathway.

JNK1 has multiple functions in cell processing, particularly in response to stress, and mediates cell apoptosis and regulates cell maturation in the gastrointestinal tract (14). Based on the induction of cell apoptosis and inhibition of cell proliferation by sulindac, we hypothesize that these functions of sulindac may be associated with increased JNK1 phosphorylation and suppression of $\beta$-catenin in human prostate cancer cells.

In conclusion, sulindac exhibits anticancer activities in human prostate cancer cells by promoting apoptosis and inhibiting cell proliferation by targeting the JNK1/ $\beta$-catenin signaling pathway. These findings indicate that sulindac may be a potential agent for prostate cancer prevention or therapy.

\section{Acknowledgements}

This study was supported in part by the Doctoral Startup Fund (grant no. 505011) from the Xinxiang Medical University (Xinxiang, China).

\section{References}

1. Siegel R, Ward E, Brawley O and Jemal A: Cancer statistics, 2011: the impact of eliminating socioeconomic and racial disparities on premature cancer deaths. CA Cancer J Clin 61: 212-236, 2011.
2. Clevers H: Colon cancer - understanding how NSAIDs work. N Engl J Med 354: 761-763, 2006.

3. Kelloff GJ, Lippman SM, Dannenberg AJ, Sigman CC, Pearce HL, Reid BJ, Szabo E, Jordan VC, Spitz MR, Mills GB, et al; AACR Task Force on Cancer Prevention: Progress in chemoprevention drug development: the promise of molecular biomarkers for prevention of intraepithelial neoplasia and cancer - a plan to move forward. Clin Cancer Res 12: 3661-3697, 2006.

4. Yang W, Bancroft L and Augenlicht LH: Methylation in the p21WAF1/cip1 promoter of Apc+/-, p21+/- mice and lack of response to sulindac. Oncogene 24: 2104-2109, 2005.

5. Yang W, Bancroft L, Liang J, Zhuang M and Augenlicht LH: p27kipl in intestinal tumorigenesis and chemoprevention in the mouse. Cancer Res 65: 9363-9368, 2005.

6. Han A, Song Z, Tong C, Hu D, Bi X, Augenlicht LH and Yang W: Sulindac suppresses $\beta$-catenin expression in human cancer cells. Eur J Pharmacol 583: 26-31, 2008.

7. Song Z, Tong C, Liang J, Dockendorff A, Huang C, Augenlicht LH and Yang W: JNK1 is required for sulindac-mediated inhibition of cell proliferation and induction of apoptosis in vitro and in vivo. Eur J Pharmacol 560: 95-100, 2007.

8. Bi X, Pohl N, Dong H and Yang W: Selenium and sulindac are synergistic to inhibit intestinal tumorigenesis in Apc/p21 mice. J Hematol Oncol 6: 8, 2013.

9. Chang L and Karin M: Mammalian MAP kinase signalling cascades. Nature 410: 37-40, 2001

10. Bode AM and Dong Z: The functional contrariety of JNK. Mol Carcinog 46: 591-598, 2007.

11. Davis RJ: Signal transduction by the JNK group of MAP kinases. Cell 103: 239-252, 2000.

12. Weston CR and Davis RJ: The JNK signal transduction pathway. Curr Opin Cell Biol 19: 142-149, 2007.

13. Weston CR, Lambright DG and Davis RJ: Signal transduction. MAP kinase signaling specificity. Science 296: 2345-2347, 2002.

14. Tong C, Yin Z, Song Z, Dockendorff A, Huang C, Mariadason J, Flavell RA, Davis RJ, Augenlicht LH and Yang W: c-Jun $\mathrm{NH} 2$-terminal kinase 1 plays a critical role in intestinal homeostasis and tumor suppression. Am J Pathol 171: 297-303, 2007.

15. Bode AM and Dong Z: Signal transduction pathways in cancer development and as targets for cancer prevention. Prog Nucleic Acid Res Mol Biol 79: 237-297, 2005.

16. Liu J and Lin A: Role of JNK activation in apoptosis: a double-edged sword. Cell Res 15: 36-42, 2005.

17. Dong C, Yang DD, Wysk M, Whitmarsh AJ, Davis RJ and Flavell RA: Defective T cell differentiation in the absence of Jnk1. Science 282: 2092-2095, 1998.

18. Kuan CY, Yang DD, Samanta Roy DR, Davis RJ, Rakic P and Flavell RA: The Jnk1 and Jnk2 protein kinases are required for regional specific apoptosis during early brain development. Neuron 22: 667-676, 1999.

19. Liu J, Minemoto Y and Lin A: c-Jun N-terminal protein kinase 1 (JNK1), but not JNK2, is essential for tumor necrosis factor alpha-induced c-Jun kinase activation and apoptosis. Mol Cell Biol 24: 10844-10856, 2004.

20. Hu D, Bi X, Fang W, Han A and Yang W: GSK3beta is involved in JNK2-mediated beta-catenin inhibition. PLoS One 4: e6640, 2009.

21. Hu D, Fang W, Han A, Gallagher L, Davis RJ, Xiong B and Yang W: c-Jun N-terminal kinase 1 interacts with and negatively regulates Wnt/beta-catenin signaling through GSK3beta pathway. Carcinogenesis 29: 2317-2324, 2008. 